\title{
Autonomous Intubation Robot System based on Visual Servoing and Hybrid Control
}

This paper was downloaded from TechRxiv (https://www.techrxiv.org).

LICENSE

CC BY 4.0

SUBMISSION DATE / POSTED DATE

01-08-2021 / 03-08-2021

\section{CITATION}

Cheng, Aohua; Qiu, Yike; Hao, Han; Xu, Yizhou; Nie, Yuxuan; Jiang, Yuhua; et al. (2021): Autonomous Intubation Robot System based on Visual Servoing and Hybrid Control. TechRxiv. Preprint. https://doi.org/10.36227/techrxiv.15087696.v1

$\mathrm{DOI}$

10.36227/techrxiv.15087696.v1 


\title{
Autonomous Intubation Robot System based on Visual Servoing and Hybrid Control
}

\author{
Aohua Cheng, Yike Qiu, Han Hao, Yizhou Xu, Yuxuan Nie, Yuhua Jiang, Xi Wu, Zhe Guo, and Gangtie Zheng
}

\begin{abstract}
During COVID-19 and other pandemics, endotracheal intubation is an effective and common method to save patients as the virus causes lung fibrosis and thus patients are unable to breathe spontaneously. Medical staff need to insert a tube close to the patient's mouth, thereby leading to a high risk of cross-infection. To protect medical staff, we propose an autonomous intubation robot system (AIRS). With the developed visual servoing and hybrid control method, the entire system can simulate doctors for satisfying repeatability and safety of intubation operations. This system includes a selfdriving/teleoperation platform, two co-robot arms, a new multifunctional laryngoscope, force sensors, and several cameras. In the visual servoing part, we realize recognition and location of the patient's face, medical devices, and main physiological structures to provide real-time navigation. In the hybrid control part, we establish an oral model, propose an offline planning method and PID controllers by combining force, vision, and motion, and apply Virtual Fixture to insert safely. AIRS's validation is with a phantom model under a 2-min operation. Our proposed robot is original and promising in the area of emergent medical robots. We will further validate AIRS in clinical applications and extend the developed techniques in other general treatments.
\end{abstract}

Index Terms-robot to combat COVID-19, endotracheal intubation, visual servoing, hybrid control.

\section{INTRODUCTION}

$\mathbf{N}$ OWADAYS, COVID-19 has been a global challenge. [1] The COVID-19 pandemic is considered the most serious global public health emergency since the end of World War II. [2] Due to the tremendous damage of COVID-19 to the lungs, numerous patients could not breathe independently. Therefore, endotracheal intubation becomes one of the most effective measures to establish effective ventilation and rescue patients. [3]

Endotracheal intubation is a surgical procedure that medical staff artificially insert a tube throughout the patient's mouth or nose to assist, or even replace their breathing. According to the operating specifications for endotracheal intubation developed

Manuscript received July 5, 2021. Part of this paper was presented at Notouch Care Workshop of IEEE ICRA 2021.

Aohua Cheng, Yike Qiu, Han Hao, Yizhou Xu, Yuxuan Nie, Yuhua Jiang, Xi Wu are undergraduate students with Digital Medicine \& Medical Robot Research Center, Tsinghua University, Beijing 100084, China (e-mail: cah18@mails.tsinghua.edu.cn;qyk18@mails.tsinghua.edu.cn; haoh19@mails .tsinghua.edu.cn;xu-yz19@mails.tsinghua.edu.cn;nyx19@mails.tsinghua.edu. cn;jiangyh@mails.tsinghua.edu.cn;wuq17@mails.tsinghua.edu.cn).

Zhe Guo a medical doctor with ICU of Beijing Tsinghua Changgung Hospital, Beijing 102218, China (e-mail: guozhe03010431@163.com).

Gangtie Zheng is professor and director of Digital Medicine \& Medical Robot Research Center, Tsinghua University, Beijing 100084, China (e-mail: gtzheng@tsinghua.edu.cn).

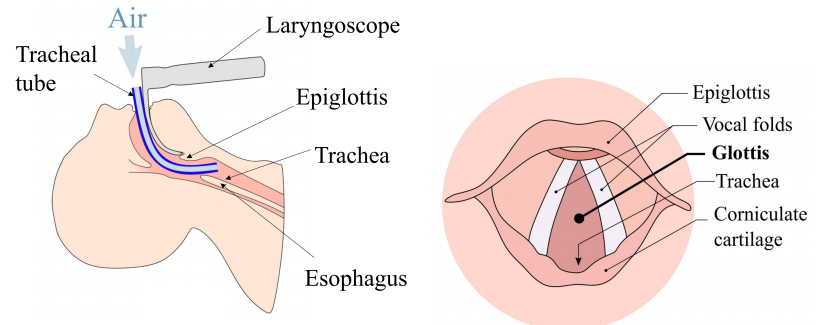

Fig. 1. Schematic diagram of general endotracheal intubation by doctors with laryngoscope lifting the epiglottis and inserting the tube through the glottis. [5]

during COVID-19, the endotracheal intubation is divided into the following main procedures: [4]

- Prepare laryngoscope, endotracheal tube, respiratory machine, anesthesia equipment (if necessary), and other materials before endotracheal intubation.

- Place patient's head in a backward position and inject muscle relaxants to keep patient sedate.

- Insert the laryngoscope into the epiglottis. Clean the oral secretions and lift the laryngoscope to expose the glottis.

- Insert the endotracheal tube into the desired airway depth through the glottis.

- Inflate the catheter balloon, connect the ventilator, and withdraw the laryngoscope.

- Make sure the patient's chest undulation and vital signs are normal.

Notably, endotracheal intubation is a high-risk procedure of emergency for cross-infection. In endotracheal intubation, doctors will operate within about $20 \mathrm{~cm}$ distance from the patient's mouth. Once the patient's airway opening, large numbers of secretions, blood, and aerosol containing the high concentrated virus will burst out into the air, which means the doctors have to take the highest level of protection. But even skilled professionals need at least 20 minutes to complete the protection wearing, which means the best time probably missed to rescue patients. [6] Therefore, considering the dilemma between emergency and safety, we hope to develop a medical robot system to conduct endotracheal intubation instead of doctors because of the popularity of robots in highrisk and repetitive scenes.

Medical robot is the most complex advanced equipment in medicine, which integrates medicine, mechanics, biomechanics, electronics, and computer science. [7] Due to its accurate operation, small trauma, and rapid recovery after 
surgery, it becomes the mainstream direction of future medical development. [8] However, most of the existing medical robots developed mainly focus on surgical applications. The emergency medical robots have not attracted enough attention from both doctors and engineers, which lacks theoretical and applied research for public health crises. Also, current medical robots are expensive and hard to learn or use by medical staff. [9] These kinds of medical robots cannot play a direct role in public health crisis response.

Although endotracheal intubation is a standard way of saving patients, conventional intubation devices still have some problems and room for improvement. Typically, they are designed only for manual operations but not for autonomous operation by a robot. The popular video laryngoscope visualizes the mouth's inner environment but with only 2D videos. [10] Moreover, an ergonomic geometric design can result in a significant impact on the practicality and smooth operation of the laryngoscope. The different ergonomic designs will produce different user experiences. [11] Such design also has important influences on the pressure exerted on patients by adjusting the size of the contact surface area and the force required to expose epiglottis. [12] Although, with the advantages of being cheaper and easier to sterilize, the traditional laryngoscopes usually require greater force to expose the epiglottis for no visual guidance, which may cause damages on patients' mouth tissue and epiglottis. On the other hand, a video laryngoscope is more expensive and difficult to disinfect, whereas is vulnerable to environmental factors and lacks three-dimensional vision. [13] Some other devices developed to improve the quality and reduce the time of the intubation process, such as REALITI and the Kepler intubation system. [5], [14] However, they still require operators close to the patients to complete the whole intubation process.

The realization of autonomously robotic intubation relies on the method and technology of autonomous visual navigation and safety control in the mouth and the trachea, which are very narrow spaces inside the human body. However, for the application to endotracheal intubation, they are not yet mature. In terms of autonomy, most of the existing surgical robots are manipulated by doctors, i.e., in the model of teleoperation, and there are few studies on autonomous robot control. From the aspect of general technology, the robotic intubation operation is similar to the scene of autonomous driving, which mainly includes visual navigation and control planning. In medicine, there are higher requirements put forward to the safety of control. However, despite the similar technology, the application scenarios are quite different. In autonomous driving, the scene is relatively large and informative. Inside a human body, the operation space is limited, complicated. Moreover, the environment may change at any time. Therefore, significant effort is still needed to realize autonomous visual navigation and safety control in the narrow space of the human body.

In this study, we aim to develop a dual-arm coordinated autonomous intubation robot system (AIRS) by simulating medical staff's operation to fight COVID-19 and protect frontline medical staff. The intubation procedure and the system design of our prototype are first presented in Section II. The navigation and control method are studied in Section III. The

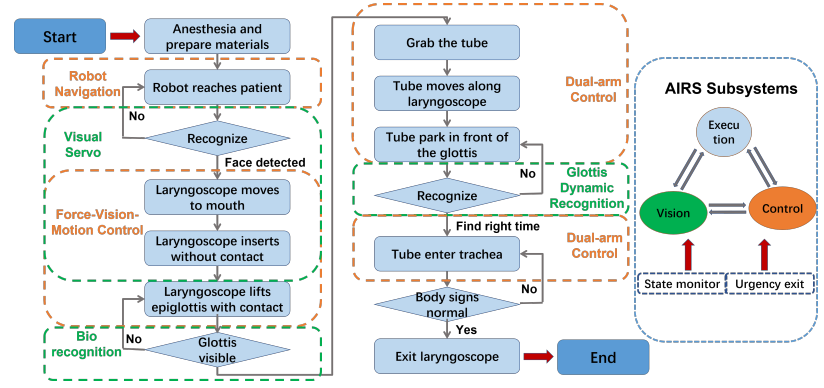

Fig. 2. Procedure and System for simulating doctors' robot autonomous intubation

experiment and results on a phantom are described in Section The conclusion and discussions are finally provided in Section $\mathrm{V}$.

\section{AUTONOMOUS INTUBATION SOLUTION}

By acquiring the requirements of frontline medical staff and discussing with ICU doctors, we first propose a robotic solution for autonomous intubation. This solution defines key components and operations by following a procedure that simulates medical standard operations. And we have finished the hardware and software implementation as AIRS' execution subsystem. The specific procedure and system design are shown in Fig. 2.

\section{A. Robotic Intubation Procedure}

After medical staff complete necessary preparations, putting the patient's head in the proper position, and moving the respiratory machine near the bed, our system begins to work. The AIRS works in the following procedure:

- The robot first reaches the patient either through automatic navigation or teleoperation.

- After the robot is in place, use the depth camera on the left arm to look for the position of the patient face.

- After the face is detected and located, move the left arm to grab the tube.

- At the same time, the right arm moves to the mouth and adjusts the posture of the laryngoscope on this arm according to the above locating information.

- The right arm inserts the laryngoscope into the mouth. During the insertion, the camera in front of the laryngoscope provides real-time depth and position estimation. Combining vision with force and motion information collected by AIRS, the laryngoscope obtains knowledge of whether it contacts the patient's mouth tissue or not.

- After the laryngoscope reaches the epiglottis, lift the epiglottis until the glottis is visible.

- Then the left arm moves the tube to the side of the laryngoscope. The tube enters against the tube slot on the laryngoscope.

- The tube parks in front of the glottis and enters the trachea at the right time.

- After checking that patient's body signs are normal, exit the laryngoscope. The intubation completes. 


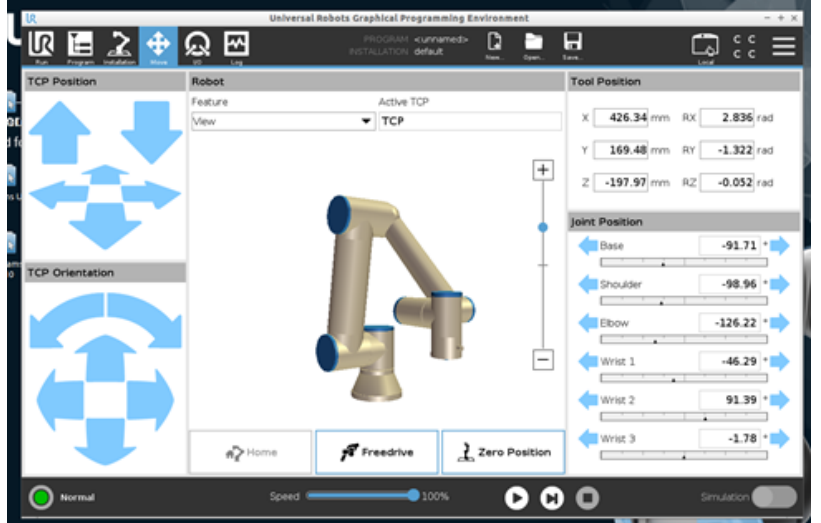

Fig. 3. State monitor interface shows the angle of each joint and allows doctors to remote-control or stop at emergency.

\section{B. System design}

1) Ideas of System Design: To simulate doctors' way of doing endotracheal intubation, the main idea of our system design is dividing the system into execution, vision, and control subsystem with reference to the functions of the doctor's hands, eyes, and brain. The vision subsystem transmits data to the control subsystem, the control subsystem issues instructions to the execution subsystem, and the execution subsystem completes the corresponding operations. These three subsystems' specific tasks show in Fig. 2 with blocks of different colors.

2) Safety Consideration: In addition, considering safety requirements, medical staff can supervise the intubation operation through state monitor and emergency exit stations in AIRS. During the intubation, the screens of the state monitor station will display videos recorded in cameras, with which medical staff can remotely monitor the operation process. In emergent situations, such as excessive force or a patient's abnormal condition, medical staff can cease the robot by the emergency exit station with an emergency stop button. Then trigger an interrupt program and withdraw the robot arm slowly from the human body.

\section{Execution Subsystem}

1) Hardware and Software Implementation: For the execution subsystem, we employed a robot platform to connect all components and devices. The platform has a battery, a 1DOF waist joint, a differential wheel automated guided vehicle $(\mathrm{AGV})$, which can move around wards by remote-control or self-navigation, a camera on the head, and two 6-DOF UR5e robot arms fixed on both sides of a robot body. The UR5e robot arm has a locating accuracy of $0.03 \mathrm{~mm}$ and a mechanical accuracy of $0.04 \mathrm{~N}$ to ensure accurate and safe operation. An OnRobot HEX-E force/torque feedback sensor and a selfdesign multi-functional laryngoscope are assembled on one of the robot arms. A Robotiq 2F-85 Gripper and an Azure Kinect DK depth camera are on the other robot arm. Our computer is equipped with an i7-10700 CPU, 16G memory, and 512G hard disk. At the same time, we also built a set of remotecontrol devices, which can control the robot and stop at any emergent time.

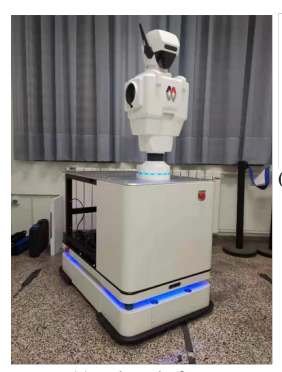

(a) Robot platform

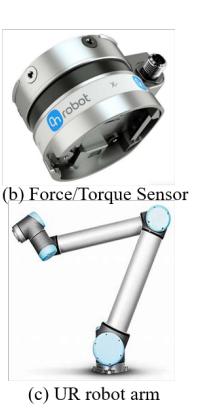

(c) UR robot arm

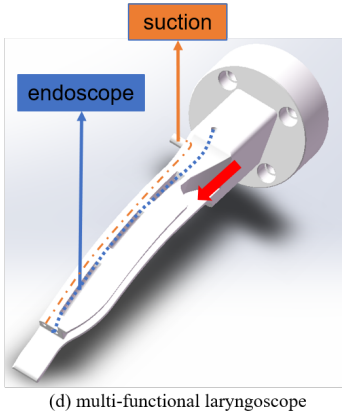

(d) multi-functional laryngoscope
Fig. 4. Key components of the system. (a) robot platform with a differential wheel AGV and robot body, (b) force/torque sensor, (c) UR5e robot arm, (d) multi-functional laryngoscope.

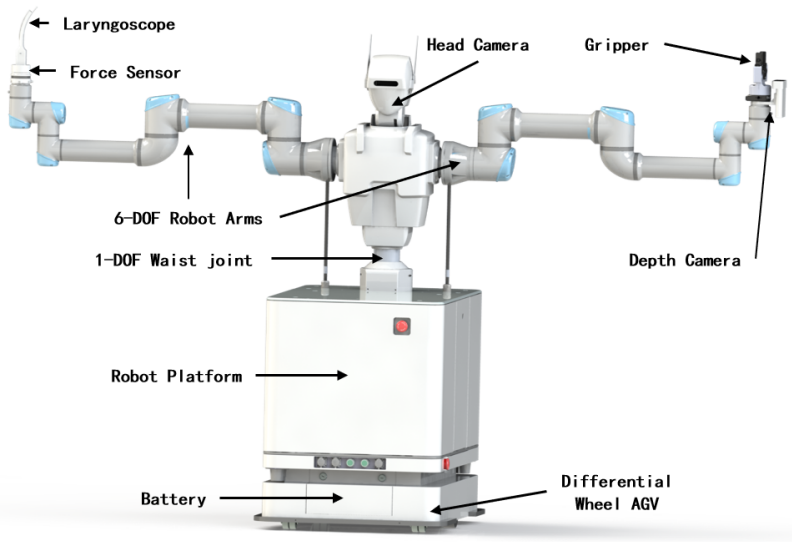

Fig. 5. The robot system with its platform, battery, AGV, waist joint, robot arms, head camera, force sensor, laryngoscope, gripper, and depth camera.

The laryngoscope is the most vital auxiliary device to complete the intubation. To make the laryngoscope suitable for the usage of the robot, we design a multi-functional laryngoscope. An endoscope is arranged at the front end of the laryngoscope structure to obtain images inside the mouth. Traditional intubation needs inserting a guidewire inside the tube before the operation, which is not convenient for both medical staff and the robot to withdraw the wire. Therefore, we design a channel on one side of the laryngoscope to guide the tube, especially when less visual information is available. To avoid the pollution of secretion, blood, and aerosol with a high concentrated virus, which burst out during this operation, to the environment, we also add the function of suction to the laryngoscope. A catheter, which connects to a sputum aspirator, is added to the device's structure, and a transparent cover can be arranged at the end of the laryngoscope to further reduce the pollution. These medical functions not only minimize the possibility of cross-infection and environmental pollution but also improve the efficiency and success rate of intubation.

\section{VISUAL AND CONTROL METHOD}

\section{A. Visual servoing}

In terms of vision subsystem, our core goal is to actively recognize and locate human face position, tube, oral physio- 


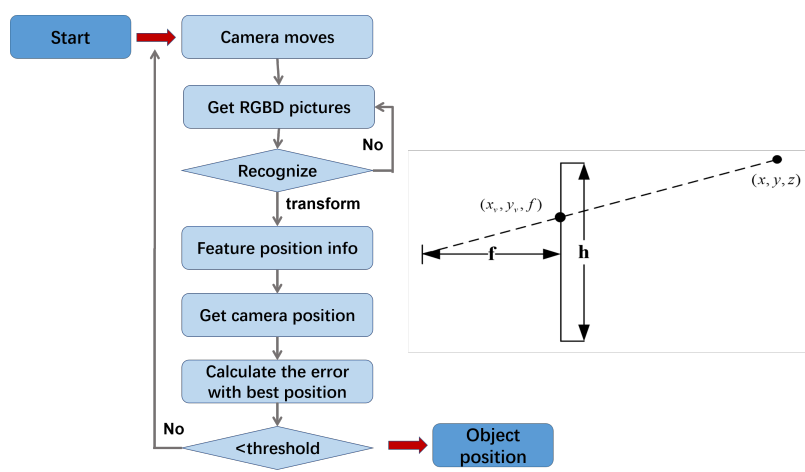

Fig. 6. Flow chart of actively recognizing and locating method using the depth camera.

logical structure and to realize oral autonomous navigation in real-time.

1) Actively Recognizing and Locating: As a basic process, we first recognize the feature points and/or lines in images, then locate the 3D coordinates relative to the robot with the depth camera. For the more accurate location, we use an active perception strategy shown in Fig. 6, which can solve the unstable accuracy of the depth camera caused by initial conditions. Taking the face as an example, we use a deep learning method [15] to get 68 feature points on the face. Then, select 4 points around the mouth and calculate the position $(x, y, z)$ of the points $\vec{F}_{i},(i=1,2,3,4)$ relative to the robot through coordinate transformation equation of the depth camera shown in Eq. 1. Then set up a mouth coordinate Oxyz by calculating the origin $\vec{O}$ and unit vector $\vec{x}, \vec{y}, \vec{z}$ of three dimensions by Eqs. 2-6 with $\overrightarrow{F_{i} F_{j}}=\overrightarrow{F_{j}}-\vec{F}_{i}$ Then we can calculate camera position $\left(x_{e}, y_{E}, z_{e}\right)$ relative to the mouth shown in Eq. 7. Finally, if the error between the current position and the best position is below the threshold, the camera will stop at its current position. If not, the error will decide the next movement of the camera. The result of face recognition and location is shown in Fig. 7a.

$$
\begin{aligned}
y= & \frac{z y_{v}}{f}, x=\frac{z x_{v}}{f}, f=\frac{h}{2 \tan (\theta / 2)} \\
\vec{O} & =\overrightarrow{F_{1}}+t \overrightarrow{F_{2} F_{1}} \\
t & =\overrightarrow{F_{3} F_{1}} \cdot \overrightarrow{F_{2} F_{1}} /\left\|\overrightarrow{F_{2} F_{1}}\right\|^{2} \\
\vec{z} & =\overrightarrow{F_{2} F_{1}} \times \overrightarrow{F_{4} F_{3}} /\left\|\overrightarrow{F_{2} F_{1}} \times \overrightarrow{F_{4} F_{3}}\right\| \\
\vec{x} & =\overrightarrow{F_{2} F_{1}} /\left\|\overrightarrow{F_{2} F_{1}}\right\| \\
\vec{y} & =\vec{z} \times \vec{x} \\
x_{e}=- & \vec{O} \cdot \vec{x}, y_{e}=-\vec{O} \cdot \vec{y}, z_{e}=-\vec{O} \cdot \vec{z}
\end{aligned}
$$

Similar to the face, feature points on the tube are also crucial for finding its location and grabbing it. Moreover, the vision subsystem needs to provide the relative position of the grasping point and the front-end point for the later control steps. The grasping point is a position of the edge in a specific proportion detected by the Hough transform. The front-end point is obtained from a machine learning method. Therefore, in the same location method of face, we can accurately grasp

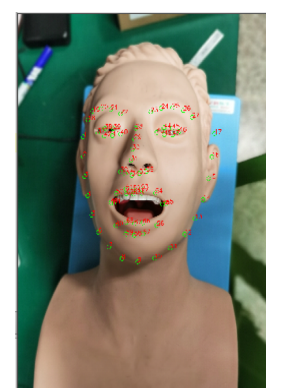

(a) Face recognition and location

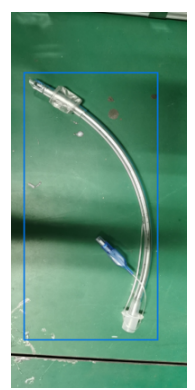

(b) Tube

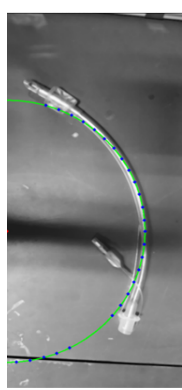

(c) Edge

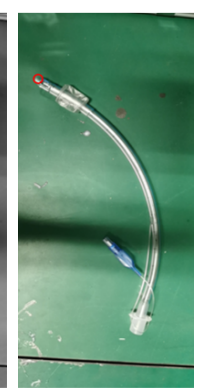

(d) Front-end point
Fig. 7. Result of recognition and location. (a) feature points of face recognition. (b-d) result of tube detection, edge detection, and front-end point detection by Hough transform

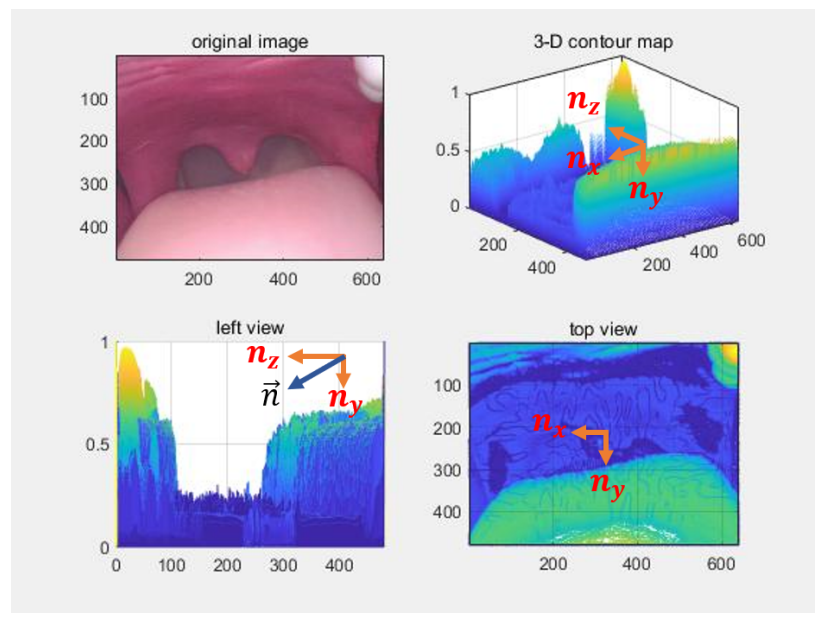

Fig. 8. Depth contour maps from different aspects of real-time navigation with the SFS estimation

the tube, initialize its posture before intubation and monitor the safety during the intubation process. The result is shown in Fig. 7.

2) Oral Autonomous Navigation: In the oral cavity, we apply the Shape From Shading (SFS) method to estimate the depth of the oral cavity in real-time, which is based on the Lambertian reflection model. [16] With this method, we can get the depth contour maps from different aspects shown in Fig. 8. Then we can estimate the front-end speed direction vector to insert the mouth.

\section{B. Hybrid control}

For the control subsystem, we focus on the safety of intubation. Since it is inevitable to contact the mouth and impossible to find an accurate oral model for all patients during the practical operation, we develop a hybrid control method by combining vision, force, and motion information.

1) Oral Model: First, we make a simplified oral model as the base of hybrid control. This oral model is expressed in a segmented function of force/torque signals from the sensors and the laryngoscope's position and posture. The segmented function is divided into four parts, i.e. the left corner, right corner, center, and tongue. In each part, we use a 


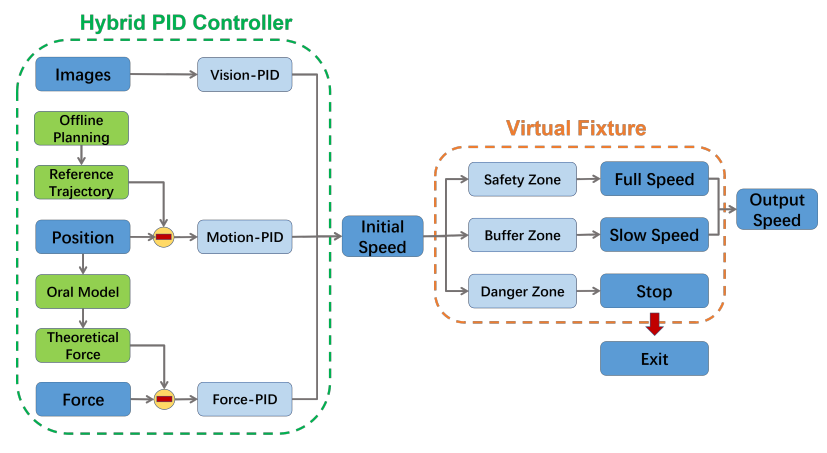

Fig. 9. Hybrid control method based on oral model, offline planning, online parallel PID, and Virtual Fixture

quadratic function as the local function. The thus formed four functions are smoothed at their junctions. The model input has seven dimensions, which are three position dimensions and four quaternion dimensions. Because the tongue is more flexible than the other parts, its deformation-force relationship is different from that of the center part with small deformation. However, considering hundreds of model parameters, we collect plenty of data with a phantom to optimize the model. To assure the data consistency each time, we use the mouth coordinate system as shown in Section III-A.

What's more, since parameter tuning for PID on real patients is slow and dangerous, the oral model makes our parameter tuning much easier and faster.

2) Offline Planning and PID Control: The offline planning process is to give the robot a reference trajectory. We use the above oral model to make a reference trajectory relative to the mouth. Each time before intubation, the depth camera locates the human mouth and computes the absolute position of the reference trajectory. We divide the trajectory into two parts. The first part is when the laryngoscope goes inside the mouth with slight contact to the mouth, and the second part is when it presses the patient's tongue to open the airway.

Since the oral environment differs from person to person, an offline trajectory might hurt the patients or finish the work imperfectly. This is why we need an online controlling system to make the robot adjust its trajectory according to different conditions.

Our online controlling algorithm is a hybrid PID controller, which parallels the PID control of vision, force, and motion. The input of the Vision-PID is the difference between the target point and the central point of the endoscope. The input of the Motion-PID is the difference between the current position and the reference trajectory. The robot reads its position in real-time and uses the oral model to compute the theoretical force in its current position. The input of the Force-PID is the difference between the actual force and the theoretical force computed by the oral model. With these three inputs, the hybrid controller will output the velocity vector.

3) Virtual Fixture: Virtual fixture is a simple direct method to judge intubation safety, which defines two hyperboloids in the seven dimension space. The two hyperboloids divide the space into the safety, buffer, and danger three zones. The robot has full speed in the safety zone, slower in the buffer zone,

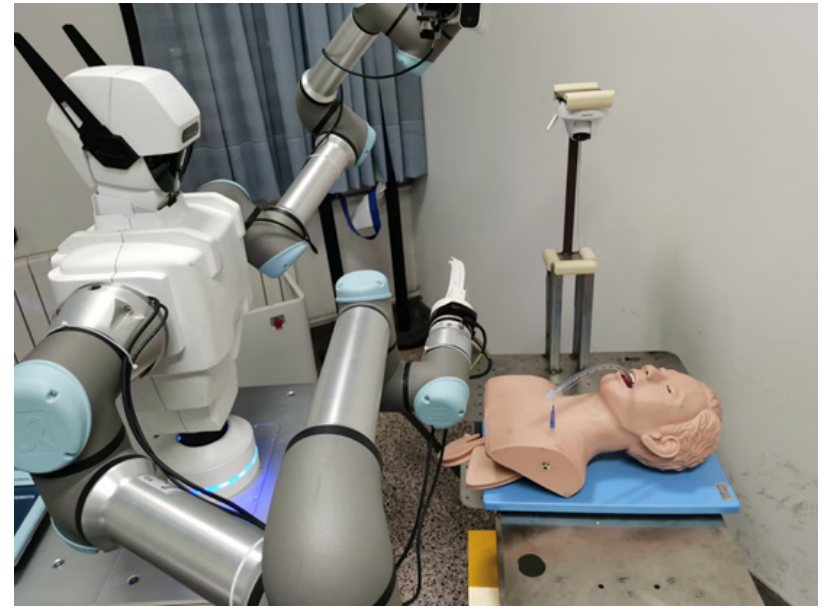

Fig. 10. The experiment platform includes a medical phantom, endotracheal tube, and AIRS, etc.

and zero in the danger zone. The danger zone will also force the robot to return along the entry trajectory at once. [17] [18] The parameters of the two hyperboloids are defined with the oral model and the experience of the doctors. The form of the virtual fixture is a simple quadratic function, which enables it to run fast. It runs in an independent thread, and at a higher frequency than the PID controller to keep the operation safe. If the hybrid control, especially the vision part, encounters an emergency like unexpected errors, Virtual Fixture can still slow down or shut down the robot to keep the patient safe.

\section{EXPERIMENT AND RESULTS ON A PHANTOM}

\section{A. Phantom experiment}

Clinical experiments are one of the core criteria for validating medical robots. We have completed the autonomous intubation experiment with a phantom in the laboratory. In the experiment, we use the standard medical phantom and endotracheal tubes to mimic practical scenes.

This experiment verifies the autonomous process from the robot arriving at the patient to exiting the laryngoscope. Specifically, the phantom experiment can divide into several steps shown in the figures below. The entire operation experiment takes about 2 minutes, which is a little bit slower than an experienced medical staff's operation time in 30 seconds to 1 minute. Experiment results indicate that both the accuracy and the safety of the operation can satisfy the requirement of practical applications

\section{CONCLUSION AND DISCUSSIONS}

\section{A. Conclusion}

As mentioned above, emergency endotracheal intubation is an important measure to rescue patients from breath dysfunction. During COVID-19 and other infectious pandemics, opening the patient airway near the medical staff makes endotracheal intubation one of the most dangerous treatments for cross-infection. Consequently, doctors need to complete the endotracheal intubation under universal protection and take turns on standby for 24 hours, which brings both physical 


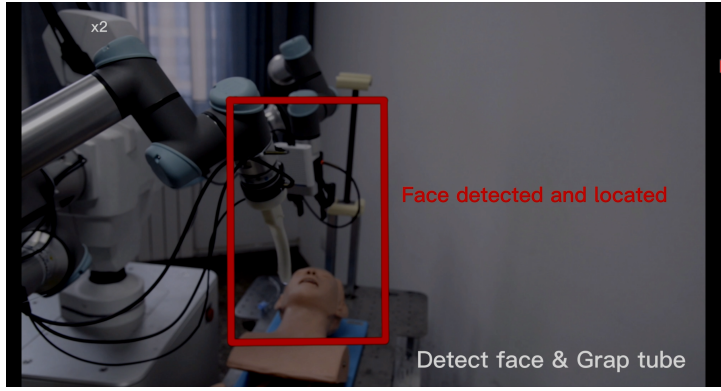

(a) Stage 1: detect face and grab tube

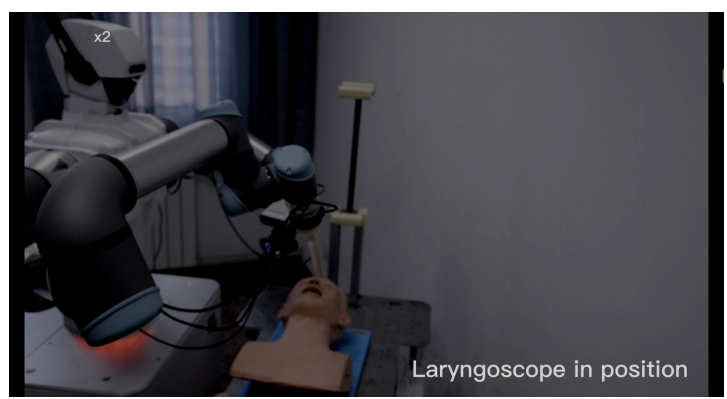

(b) Stage 2: the laryngoscope arrives at the mouth

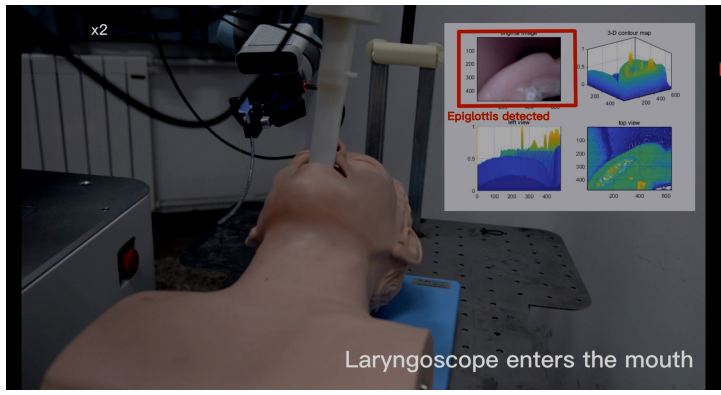

(c) Stage 3: the laryngoscope enters the mouth

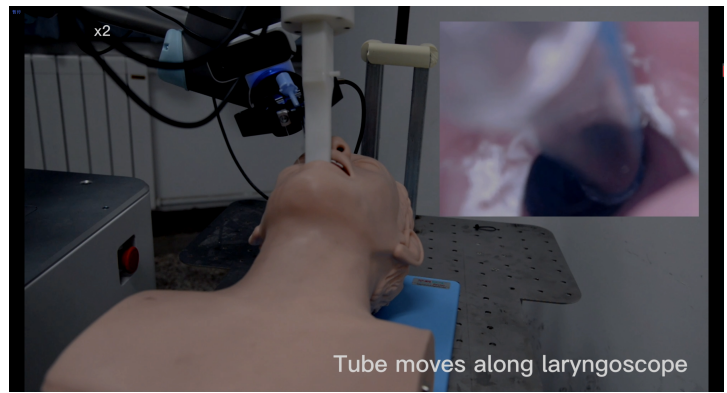

(d) Stage 4: the tube moves along the laryngoscope

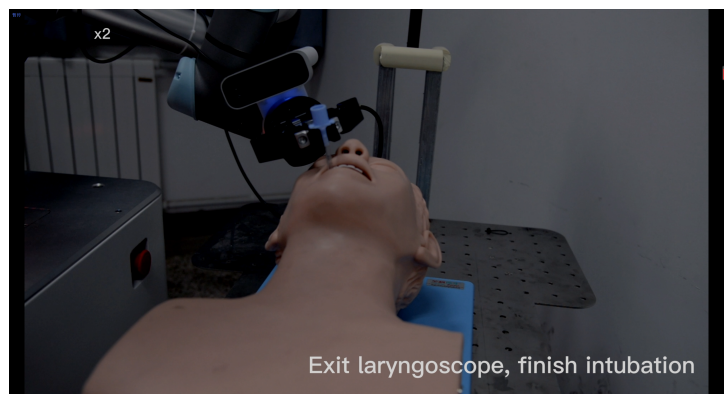

(e) Stage 5: exit laryngoscope and finish the entire intubation

Fig. 11. Main stages of autonomous endotracheal intubation by AIRS. and psychological pressure to medical staff. To solve this dilemma between patients' intubation and doctors' protection, we propose this dual-arm coordinated AIRS to replace medical for such risk repetitive intubation and explore new non-contact treatment in the post-pandemic era.

We comprehensively consider the hardware and software design with the requirements of frontline medical staff, which leads to our three subsystems: execution, vision, and control to simulate the practical medial operations. In the execution subsystem, we established the whole robot platform and designed a new multi-functional laryngoscope suitable for robot intubation. In the vision subsystem, we realized feature recognition, location, and navigation in such a narrow space as the mouth. In the control subsystem, we applied visual path planning and force-vision-motion hybrid control to guarantee safety based on general oral structure. By and large, our autonomous intubation system can release doctors' pressure and make the operation smarter and safer.

After preliminary system construction and experimental verification with standard medical phantom, we plan to conduct animal and clinical experiments along with further optimization of the robot. In particular, we plan to conduct experiments first on pigs as they are commonly used in medical device experiments.

Similarly, as the standard procedure of developing a medical robot, after completing the animal experiments, we will conduct the final verification experiment on the human body. Since the human is under anesthesia, we can deem patients to be quasi-static as in phantom and animal experiments.

As Covid-19 is still raging across the world, with the idea, methodology, and techniques, we hope that this report can provide help to fighting the virus and protecting frontline medical staff.

\section{B. Discussion}

This project has a wide range of worth improvements and application scenarios, including but not limited to the following points.

1) General Endotracheal Intubation: Although after COVID-19, there will not be many patients needing intubation, the application of endotracheal intubation in medical treatment is still ubiquitous. For example, an anesthesiologist must first complete the intubation operation for the patient breathing maintenance in most surgery. Therefore, if there are devices to replace the doctor for intubation operation, it can not only improve the surgical efficiency, but also the safety in avoiding any risk of infection from any unknown infectious virus. With the added function of a sputum aspirator and a transparent cover to the laryngoscope, safety can be significantly enhanced.

2) Intubation Teaching Aids: In traditional intubation training, doctors usually can only teach empirically through words or practices but without accurate instruction, which leads to the intubation success rate often depending on doctors' experience. Using the mechanical and visual information recorded by AIRS, we can reproduce the operating procedures and sensor information. By analyzing and recording a large amount of data, we can obtain more general and instructive medical rules 
and advice, which helps interns have a more vivid surgical experience and enhances their skills.

3) Tele-endotracheal Intubation: Traditional telemedicine mostly stays at a low level like teleconsultation. However, because AIRS can realize non-contact intubation, the intubation operation can be completed under the doctors' observation only by setting a monitor window. If some remote-control devices can be developed, automatic and teleoperation intubation mode can be realized remotely. Therefore, people in economically underdeveloped areas can obtain the same quality medical service as those in the well-developed area with lower costs, which is of great social value and significance to our society.

4) Multifunctional Medical Robot: Traditional surgical robots usually only have a single function. However, AIRS can easily expand other functions like temperature measurement, oropharyngeal swab sampling, and other universal operations based on similar technical methods, which means that AIRS has great potential for versatility and popularization.

\section{ACKNOWLEDGMENT}

The authors thank Beijing Tsinghua Changgung Hospital, Beijing, China for providing professional suggestions and requirements. The authors also thank the Spring Foundation from Tsinghua Education Fund, Beijing, China for main financial support.

\section{REFERENCES}

[1] T. P. Velavan and C. G. Meyer, "The covid-19 epidemic," Tropical medicine \& international health, vol. 25, no. 3, p. 278, 2020.

[2] Y. Shi, G. Wang, X.-p. Cai, J.-w. Deng, L. Zheng, H.-h. Zhu, M. Zheng, B. Yang, and Z. Chen, "An overview of covid-19," Journal of Zhejiang University. Science. B, p. 1, 2020.

[3] B. A. Orser, "Recommendations for endotracheal intubation of covid-19 patients," Anesth Analg, vol. 130, no. 5, pp. 1109-1110, 2020.

[4] W. Yao, T. Wang, B. Jiang, F. Gao, L. Wang, H. Zheng, W. Xiao, S. Yao, W. Mei, X. Chen et al., "Emergency tracheal intubation in 202 patients with covid-19 in wuhan, china: lessons learnt and international expert recommendations," British journal of anaesthesia, vol. 125, no. 1, pp. e28-e37, 2020.

[5] Q. Boehler, D. S. Gage, P. Hofmann, A. Gehring, C. Chautems, D. R. Spahn, P. Biro, and B. J. Nelson, "Realiti: A robotic endoscope automated via laryngeal imaging for tracheal intubation," IEEE Transactions on Medical Robotics and Bionics, vol. 2, no. 2, pp. 157-164, 2020

[6] L. Meng, H. Qiu, L. Wan, Y. Ai, Z. Xue, Q. Guo, R. Deshpande, L. Zhang, J. Meng, C. Tong et al., "Intubation and ventilation amid the covid-19 outbreak: Wuhan's experience," Anesthesiology, vol. 132, no. 6 , pp. $1317-1332,2020$.

[7] B. S. Peters, P. R. Armijo, C. Krause, S. A. Choudhury, and D. Oleynikov, "Review of emerging surgical robotic technology," Surgical endoscopy, vol. 32, no. 4, pp. 1636-1655, 2018.

[8] C. Freschi, V. Ferrari, F. Melfi, M. Ferrari, F. Mosca, and A. Cuschieri, "Technical review of the da vinci surgical telemanipulator," The International Journal of Medical Robotics and Computer Assisted Surgery, vol. 9, no. 4, pp. 396-406, 2013.

[9] C. Ho, E. Tsakonas, K. Tran, K. Cimon, M. Severn, M. MierzwinskiUrban, J. Corcos, and S. Pautler, "Robot-assisted surgery compared with open surgery and laparoscopic surgery: Clinical effectiveness and economic analyses," 2011. [Online]. Available: http://europepmc.org/ books/NBK168912

[10] B. Shippey, D. Ray, and D. McKeown, "Use of the mcgrath ${ }^{\circledR}$ videolaryngoscope in the management of difficult and failed tracheal intubation," British journal of anaesthesia, vol. 100, no. 1, pp. 116-119, 2008.
[11] L. Bartolomeo, Y. Noh, Y. Kasuya, M. Nagai, M. Zecca, S. Sessa, S. Cosentino, K. Saito, Z. Lin, H. Ishii et al., "Biomechanical evaluation of the phases during simulated endotracheal intubation (eti): Pilot study on the effect of different laryngoscopes," in 2013 35th Annual International Conference of the IEEE Engineering in Medicine and Biology Society (EMBC). IEEE, 2013, pp. 4887-4890.

[12] S. Hashimoto, S. Manabe, S. Kaku, T. Wang, and R. Tanaka, "Measurements of pressure between laryngoscope blade and upper airway tract during orotracheal intubation," in Proceedings of the First Joint BMES/EMBS Conference. 1999 IEEE Engineering in Medicine and Biology 21st Annual Conference and the 1999 Annual Fall Meeting of the Biomedical Engineering Society (Cat. N, vol. 1. IEEE, 1999, pp. 330-vol.

[13] V. Nambiraj, M. Chinnaraman, N. Viswanathan, and A. Mahimairaj, "Design of low-cost customized laryngoscope," in 2019 IEEE International Conference on Intelligent Techniques in Control, Optimization and Signal Processing (INCOS). IEEE, 2019, pp. 1-5.

[14] T. Hemmerling, R. Taddei, M. Wehbe, C. Zaouter, S. Cyr, and J. Morse, "First robotic tracheal intubations in humans using the kepler intubation system," British journal of anaesthesia, vol. 108, no. 6, pp. 1011-1016, 2012.

[15] I. Lenz, H. Lee, and A. Saxena, "Deep learning for detecting robotic grasps," The International Journal of Robotics Research, vol. 34, no. 4-5, pp. 705-724, 2015.

[16] R. Zhang, P.-S. Tsai, J. E. Cryer, and M. Shah, "Shape-from-shading: a survey," IEEE transactions on pattern analysis and machine intelligence, vol. 21, no. 8, pp. 690-706, 1999.

[17] Y. He, Y. Hu, P. Zhang, B. Zhao, X. Qi, and J. Zhang, "Human-robot cooperative control based on virtual fixture in robot-assisted endoscopic sinus surgery," Applied Sciences, vol. 9, no. 8, 2019. [Online]. Available: https://www.mdpi.com/2076-3417/9/8/1659

[18] I. Lenz, H. Lee, and A. Saxena, "Deep learning for detecting robotic grasps," The International Journal of Robotics Research, vol. 34, no. 4-5, pp. 705-724, 2015. [Online]. Available: https: //doi.org/10.1177/0278364914549607 